\title{
Design of technology-based rehabilitation pathways: the experience of Santobono-Pausilipon Hospital
}

\author{
L. Iuppariello ${ }^{1}$, M. Cesarelli ${ }^{2}$, G. Faiella ${ }^{3}$, S. Esposito ${ }^{1}$, M. Nespoli ${ }^{1}$, L. Foggia ${ }^{1}$, F. Clemente ${ }^{4,3}$ \\ ${ }^{1}$ Department of Rehabiliation, AORN Santobono-Pausilipon, Naples, Italy \\ 2 Department of Electric Engineering and Information Technologies (DIETI), School of Engineering, University of Naples Federico II, Naples, \\ Italy \\ ${ }^{3}$ Fondazione Santobono Pausilipon, Naples, Italy \\ ${ }^{4}$ Institute of Cristallography, Italian National Research Council (IC-CNR), Rome, Italy
}

\begin{abstract}
The fields of rehabilitation robotics and virtual reality are becoming a growing area of interest in the clinical rehabilitation of people with motion impairments. These systems have the potential to assess abilities through physiological measurements and modelling activities, such as posture, gait, and balance. They can also be used as rehabilitative tools by providing patients with task-specific training in a motivating and engaging way. Despite the potential advantages of such systems, until now, there has been a general limitation of their use in rehabilitative practice. Robotics and virtual reality systems can be challenging, engaging, and fun all at the same time, particularly for children with disabilities (since they are often not very motivated to comply with conventional therapies). The aim of this work is to accurately describe the clinical use of innovative rehabilitative technologies and their use in the development of two technology-based rehabilitation pathways for the treatment of gait disorders following obesity and neurological diseases in the treatment of pediatric patients.
\end{abstract}

\section{Section: RESEARCH PAPER}

Keywords: physiological measurement; rehabilitation; clinical pathways; virtual reality; gait analysis; health management

Citation: L. Iuppariello, M. Cesarelli, G. Faiella, S. Esposito, M. Nespoli, L. Foggia, F. Clemente, Design of technology-based rehabilitation pathways: the experience of Santobono-Pausilipon Hospital, Acta IMEKO, vol. 7, no. 4, article 10, December 2018, identifier: IMEKO-ACTA-07 (2018)-04-10

Editor: Alexandru Salceanu, "Gheorghe Asachi" Technical University of lasi, Romania

Received March 30, 2018; In final form September 21, 2018; Published December 2018

Copyright: (C) 2018 IMEKO. This is an open-access article distributed under the terms of the Creative Commons Attribution 3.0 License, which permits unrestricted use, distribution, and reproduction in any medium, provided the original author and source are credited

Corresponding author: Luigi luppariello, e-mail: I.iuppariello@santobonopausilipon.it

\section{INTRODUCTION}

The concept of patient-based rehabilitation is a crucial issue for every healthcare provider. Its aim is to overcome a diseasebased approach to healthcare that often does not consider patients' preferences and their emotional needs and life issues. Assistive technology-based rehabilitation is an area of research relating to the use of technology within the realm of physiotherapy [1-3]. Whatever the cause of impairment, the main aim of most neurological rehabilitation is to facilitate the learning or relearning of motor skills. The process of gaining motor skills involves repeating the same movement hundreds or even thousands of times. Traditional rehabilitation is time consuming and requires lengthy sessions with therapists that are demanding on personnel and healthcare budgets. Patients often tire of rehabilitation, finding it frustrating or dull. Integrating virtual, augmented, and mixed reality environments into rehabilitation protocols has the potential to change the nature of rehabilitation, particularly in the neurorehabilitation field [4-5].

In recent years, beyond conventional physical treatment, various new rehabilitation strategies, such as robotics and virtual reality (VR), have been introduced and proven effective in improving walking performance. Disabilities, residual motor function, and efficacy of treatment are often quantified using semi-quantitative evaluation scales, without reliable quantitative measures. Especially in the field of rehabilitation medicine, robotics and VR systems have the potential to objectively measure abilities and to serve as therapeutic tools. They provide users with repetitive, contextualized, task-specific training in an exciting and entertaining manner. Such exercises are fundamental in neurorehabilitation as they aid in promoting neuroplasticity and recovery. Robotic systems can facilitate or assist the movement of paretic limbs in performing functional movements and actions, enabling patients to increase their social engagement and therefore their autonomy and independence. 
VR systems are based on three-dimensional computer-generated simulations of the real world. They offer a variety of mechanisms for therapeutic gain, including repetitive practice of movements; engagement in problem-solving, memory, and attention tasks; and exposure to anxiety-provoking stimuli or events. Moreover, innovative computerized systems can define physiological models of the human body and provide a quantitative measure of biomechanical features of human movements. This is useful for quantitatively assessing baseline motor conditions; individually adapting the rehabilitation program with residual motor function and treatment objectives; and measuring improvements. VR systems, for instance, offer real-time feedback regarding performance that can be provided to patients and clinicians (which is useful for addressing the rehabilitation targets) by monitoring changes during the rehabilitation pathways (reports can be produced to provide objective data regarding patient progress in comparison to themselves or other users).

The appropriate use of robotics and VR systems [6] can be challenging, engaging, and fun all at the same time, particularly for children with disabilities, who are often not very motivated to comply with conventional therapies that they do not think are meaningful or fun. Moreover, the use of VR systems in pediatric patients is suitable thanks to the high plasticity of neuronal circuits in children. Despite these advantages, the practical introduction of new technologies requires introducing an ad hoc quality control management plan for multidimensional and multidisciplinary procedures (i.e., new clinical pathways) in the clinical system.

Clinical pathways are predefined, articulated, and coordinated sequences of services provided at the outpatient, hospitalization, and/or geographical level. They involve the integrated participation of various specialists and professionals (in addition to the patient themselves) at the hospital and/or geographical level in order to achieve the most appropriate diagnosis and therapy for a specific pathological situation. They are specifically recommended by the Italian National Health Service with the aim of guaranteeing the essential levels of healthcare [7].

Most traditional rehabilitative pathways are performed in a passive way, without a patient-centered approach; consequently, improvements of the patients' health cannot be maintained [811]. To overcome this limitation, this paper introduces specific patient-centered pathways, designed using an innovative standard of patient flow through the healthcare system [12].

The aim of this work is to describe the clinical implementation of two technology-based rehabilitation pathways in the Rehabilitation Unit of the pediatric hospital SantobonoPausilipon in Naples, Italy. This paper includes (i) a description of the technologies; (ii) an explanation of the implementation of the new standardized procedures based on innovative physiological measurements and activities; and (iii) the main results from the clinical implementation of the procedures.

\section{MATERIALS}

The foundation of a successful rehabilitation program and its monitoring is a well-developed plan of action based on the specific limitations of each patient. Even if two persons have the same physical limitation or the same disease, different circumstances may impair one more than the other. Therefore, it is necessary to construct a personalized and optimized rehabilitation program for each patient. With this consideration in mind, the Rehabilitation Unit of the pediatric hospital
Santobono-Pausilipon in Naples, Italy has developed several individual rehabilitation pathways combining conventional physiotherapy with robotics and VR systems for the treatment of children with gait disorders. The following robotic and virtual reality systems have been used:

- Gait Real-Time Analysis Interactive Lab (GRAIL), for both gait analysis (baseline and follow-up evaluations) and rehabilitation.

- Lokomat, Erigo, and AlterG systems for motor rehabilitation.

These technologies have been used to flank and support clinicians in the implementation of two technology-based rehabilitation pathways in the treatment of gait disorders following neurological diseases and obesity in childhood.

In the following sections, the technologies are described in detail.

\subsection{GRAIL}

\subsubsection{Architecture}

GRAIL [5], produced by Motek Medical, has shown encouraging prospects in terms of its evaluative and rehabilitative benefits. GRAIL offers clinical gait analysis and gait training using the latest technologies, which are solidly integrated into one functional system, providing the full functionality of a traditional gait lab and more. It can be used as a standard for a variety of patient groups, including those with neurological, orthopedic, and muscular-skeletal complaints and elderly persons with an increased risk of falling. The architecture consists of a self-paced instrumented dual-belt treadmill, an integrated motion-capture system, and synchronized VR environments next to three video cameras. The entire GRAIL setup can be classified into three categories: i) "system operating area," ii) "operator desk," and iii) "background processing" (Figure 1). The "system operating area" includes all the hardware with which the subject has direct interaction. Almost all components in this part are present in order to create an immersive experience for the subject. The "operator desk" includes all the hardware with which the operator has direct interaction. It includes two computers (D-Flow and MoCap) to manage the entire system. The D-Flow computer uses two dedicated monitors, one mouse and one keyboard. The operation of the D-Flow computer is like that of any other PC. The DFlow software controls the whole system, managing the relationship between the subject, the scenario, and the interactive feedback and simulations [13]. As well as the D-Flow computer, GRAIL includes many other computers. These computers are all connected to one monitor, one mouse, and one keyboard. To control all computers with one set of monitors, a mouse, and a

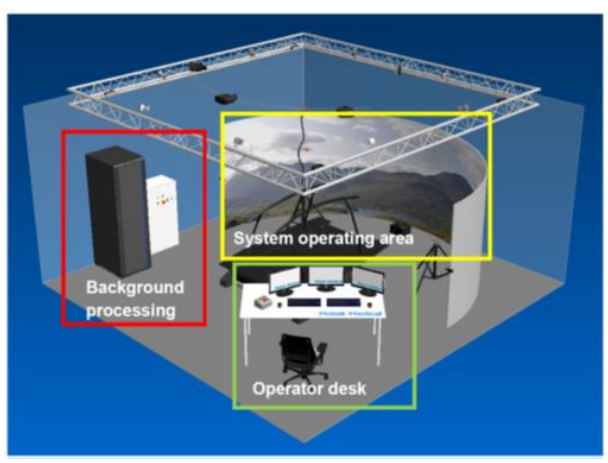

Figure 1. The GRAIL system. 


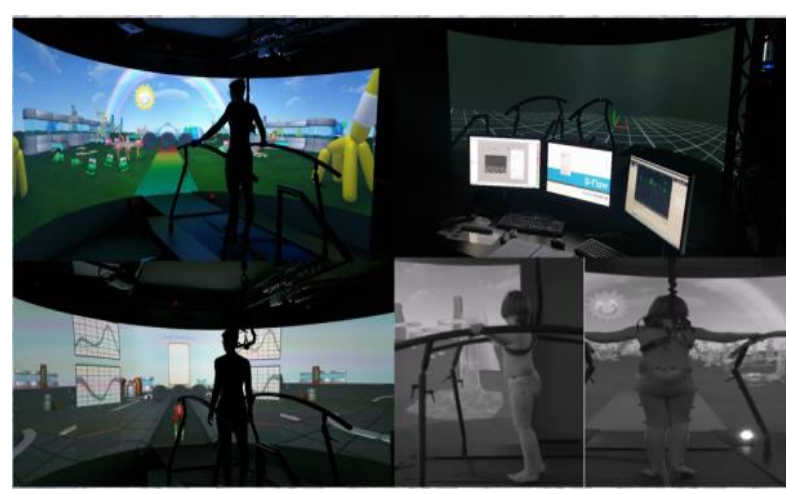

Figure 2. Functional gait analysis using the GRAIL system.

keyboard, a KVM switch is used. From here, the operator controls what happens in the "system operating area." Finally, "background processing" includes all the hardware that is needed to control the system.

This system permits the calculation of all gait parameters in real time (spatio-temporal parameters, kinematics, kinetics, and muscle-activation). The self-paced mode permits the subject to walk at a self-selected speed, while the treadmill and the VR environment are perfectly synchronized. Accurate gait parameters are ensured due to a natural walking pattern, facilitated by an immersive VR environment projected onto a $180^{\circ}$ screen. This gives the subject the impression of walking in a natural environment with a peripheral view, while measuring gait behavior, thereby providing a "functional gait analysis" (Figure 2). The accuracy of the data can be verified in real time. An offline analytical tool offers dedicated data analysis.

\subsubsection{Calibration}

A GRAIL gait analysis session requires appropriate preparation with additional steps in the motion capture software and D-Flow. First, the motion capture system and the force plates need to be calibrated. Two steps are commonly involved in the motion capture system calibration: static calibration and camera calibration. Static calibration is performed by using a wand embedded with five markers emitting red LED lights. This step allows us to set the camera position and the global origin of the acquisition volume where the subjects will be analyzed. Camera calibration involves moving the wand in the acquisition volume so that each camera collects the required number of refinement frames in the acquisition volume. Finally, the force plates are calibrated with a specific module on the D-Flow computer, when no weight is exerted on them.

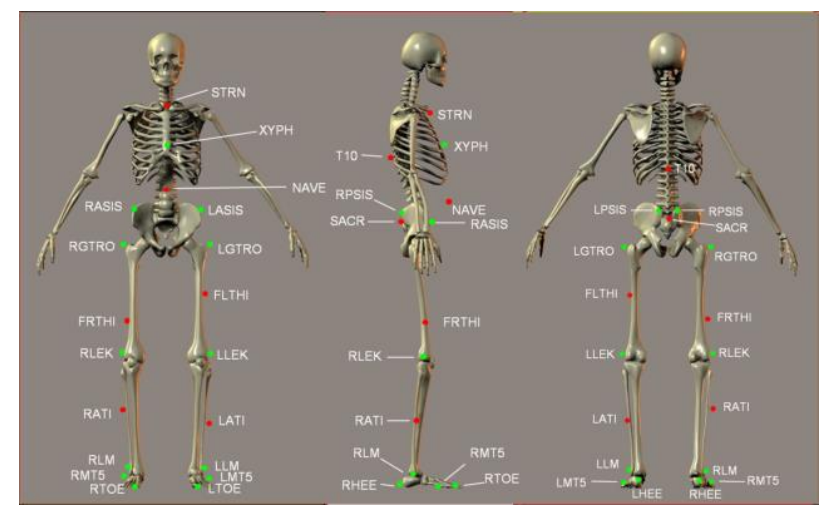

Figure 3. The position of the markers on the body subject for gait analysis and gait training sessions.

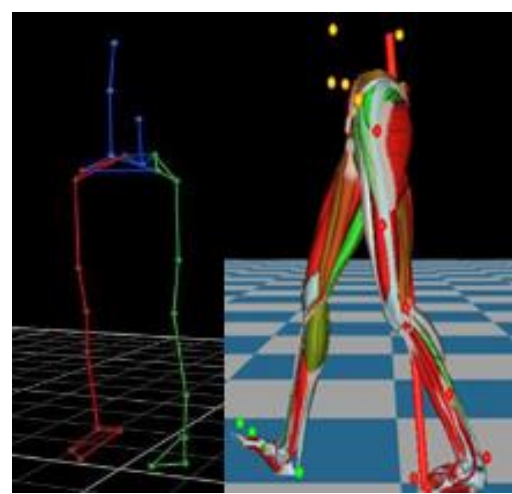

Figure 4. The virtual skeleton (on the left) and the full human body model.

\subsubsection{Gait analysis and gait training using GRAIL}

Once the calibrations of both the motion capture system and the force plates are complete, the system is ready to perform a gait analysis session. Reflective markers are placed on the subject's skin (Figure 3) and the Cartesian position of each marker relative to the global coordinate system is tracked using high-speed infrared cameras.

Before starting the physical task, the subject performs a Range of Motion (ROM) trial on the treadmill to minimize real-time labelling errors and to create the virtual skeleton or full human body model (HBM), as shown in Figure 4.

The HBM uses inverse dynamics to estimate muscle forces and visualize them in a virtual environment in real-time. It allows for both the visualization and calculation of muscle forces, joint angles, moments, and powers in real time during the gait sessions. The subject measurements as well as the marker and force plate data are input into the model. Inverse dynamics are used to calculate the biomechanical outputs.

The HBM can be displayed on the screen to represent the patient in the VR environment, and as muscles are activated, the muscles light up and change color depending on the amount of force applied. The patient walks on the treadmill at either a fixed, comfortable speed that can be adjusted by the operator or in a self-paced mode (i.e., the treadmill speed is controlled by the patient's position on the treadmill). The operator sets a VR scene that is a futuristic, colorful, non-distracting environment.

The ability of the GRAIL system to simulate real-life learning while providing augmented feedback and a high intensity of massed practiced tasks can be used for a variety of patient groups with neurological or orthopedic disorders. It can help in the development of technology-based rehabilitation pathways in which a multidisciplinary team composed of neurologists, rehabilitation specialists, orthopedists, engineers, and physiotherapists can collaborate with one another to both evaluate patients' functional behaviors and help restore or improve that function.

\subsection{Lokomat}

The Lokomat (Figure 5) is a robotic treadmill training system that uses augmented biofeedback and a body weight support system to suspend individuals while their legs are attached to robotic legs that assist them with basic walking functions.

It allows the therapist to control how fast the patient walks, how much body weight the patient can support, and how much assistance the robotic legs give the patient through a range of motions. The customized support and limb guidance provided by the Lokomat allows patients who have a wide variety of 


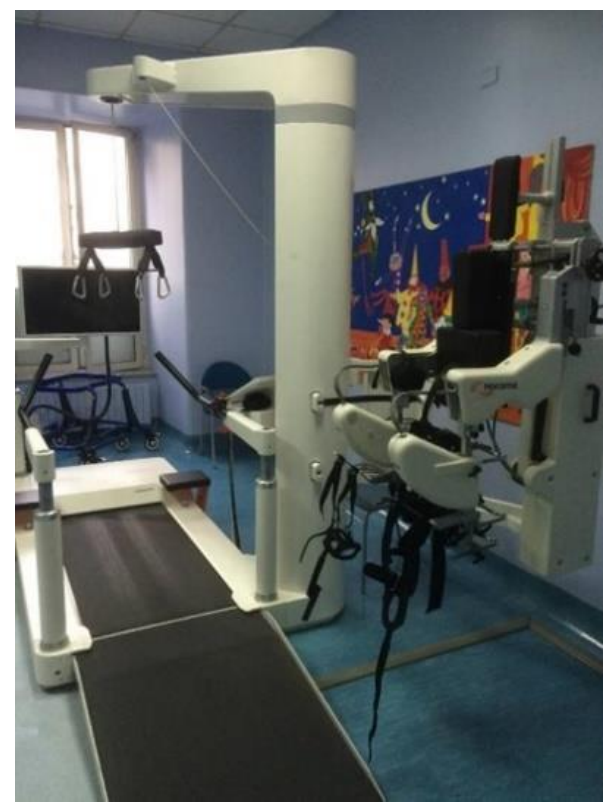

Figure 5. The driven gait orthosis Lokomat guides the legs of the subject walking on the treadmill. A body weight support system further facilitates the walking movements.

walking impairments to use this device both in inpatient and outpatient settings.

It is used to improve mobility in individuals following a stroke, a spinal cord injury, a traumatic brain injury, multiple sclerosis, cerebral palsy, and other neurological diseases and injuries.

\subsection{Erigo}

The Erigo (Figure 6) is a robotic system used in the rehabilitation of neurological patients. It accelerates the recovery

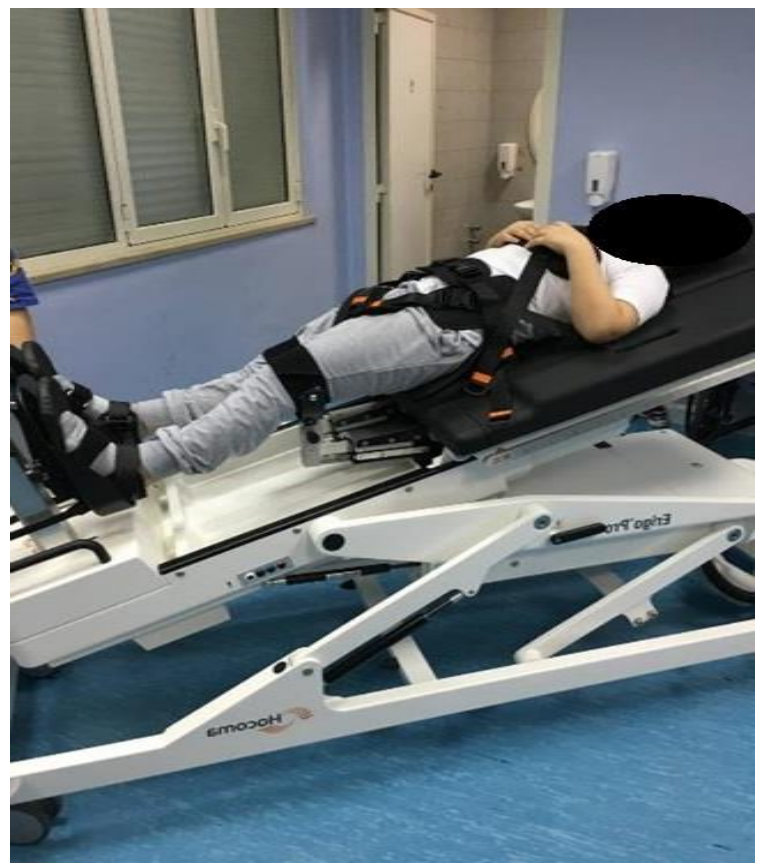

Figure 6. The ERIGO device combines progressive verticalization and cyclic leg movements in combination with step-synchronized muscle functional electric stimulation at the lower limb level to ensure safe stabilization when the patient is upright.

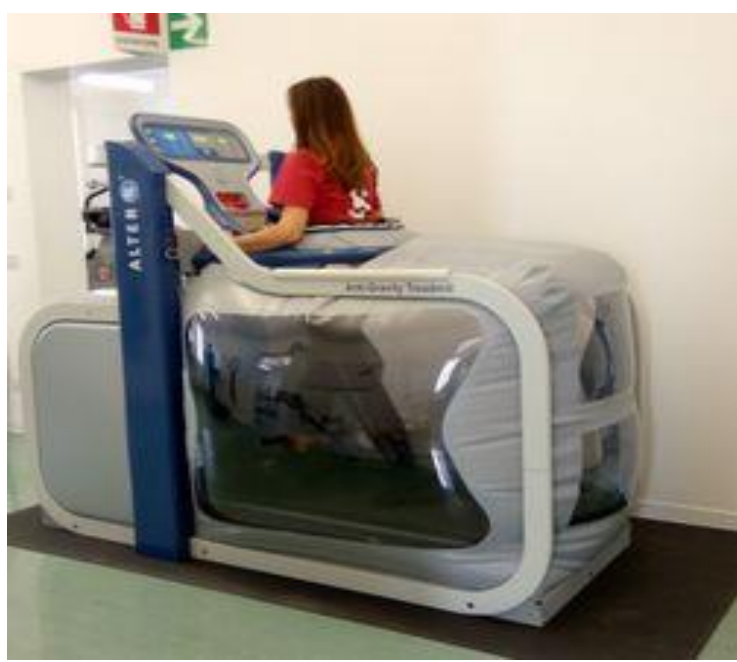

Figure 7. The AlterG anti-gravity treadmill. It uses differential air pressure technology to provide lower body positive pressure unweighting.

process with intensive sensorimotor stimulation and prevents potential damage due to immobilization.

\subsection{AlterG}

The AlterG is a treadmill (Figure 7) formed of two compressors, a protective transparent chamber, safety locks, and different sizes of pants to hold the patient firmly in the chamber.

The patient can walk without bearing their entire weight, thereby reducing the impact on the body, in turn optimizing rehabilitation and physical therapy outcomes. Its Differential Air Pressure (DAP) technology applies a lifting force to the body that reduces the weight on the lower extremities and allows for precise unweighting of up to $80 \%$ of a person's body weight, finding exactly where the pain stops and making natural movement feel good again.

\section{METHODS}

The use of technology-based rehabilitation pathways has clear positive effects on the safety and quality of care, particularly for children, thereby improving their motor function outcomes and disability.

These considerations led the pediatric hospital SantobonoPausilipon to define and approve two new rehabilitation models of care combining different robotics and VR systems for the treatment of patients suffering from neurological diseases and obesity. The idea underpinning the models is to construct two organized, goal-defined, and time-specified plans of management of the gait disorders in which a multidisciplinary team of clinicians and engineers works together to achieve better quality outcomes by applying new technologies. In this way, children progress along a technology-based pathway designed according to the healthcare standards of the Italian Ministry of Health and the Regional Health System.

\subsection{Neurological diseases}

At the Santobono-Pausilipon Hospital, the only pediatric rehabilitation center for post-acute treatments (cod.56 of the Regional Health System) and brain injuries (cod.75 of the Regional Health System) has recently been opened, according to the Regional Plan of Programming of the Hospital Network. The mission of this center is to guarantee global care for children with severe diseases. Participants in this study were recruited from both the inpatient and outpatient settings of the Rehabilitation 
Unit of the pediatric hospital Santobono-Pausilipon. For inclusion in the study, the participants were required to have neurological diseases (ataxia, post-stroke spasticity, cerebral palsy, etc.) associated with gait disorders. The study excludes those who are unable to walk without direct assistance or those who have major cognitive deterioration that would impact participation. All the patients were enrolled in the study by clinicians. Further information was given to the parents of those satisfying the entry criteria, and they were asked to declare their willingness for their children to comply with whichever treatment option to which they are assigned and to attend all the follow-up visits.

The baseline and follow-up evaluations consist of traditional clinical scales (i.e., the Gross Motor Function Measure, the Berg Scale), and gait analysis was assessed using GRAIL (Figure 8). Inpatient and outpatient children, depending on their residual motor capabilities, completed gait training sessions using the Erigo, Lokomat, AlterG, and GRAIL.

\subsection{Obesity}

The rehabilitation pathways for children suffering from obesity have been developed with the aim of achieving improvements in postural balance, gait, and the reduction of excess weight or fat mass. These children were recruited from an outpatient setting (Day Hospital Rehabilitation) of the Children Obesity and Endocrine Disorders Unit of the pediatric hospital Santobono-Pausilipon. For inclusion in the study, the participants were simply required to be suffering from obesity. The study excludes those who are unable to walk without direct assistance or those who have major cognitive deterioration that would impact participation.

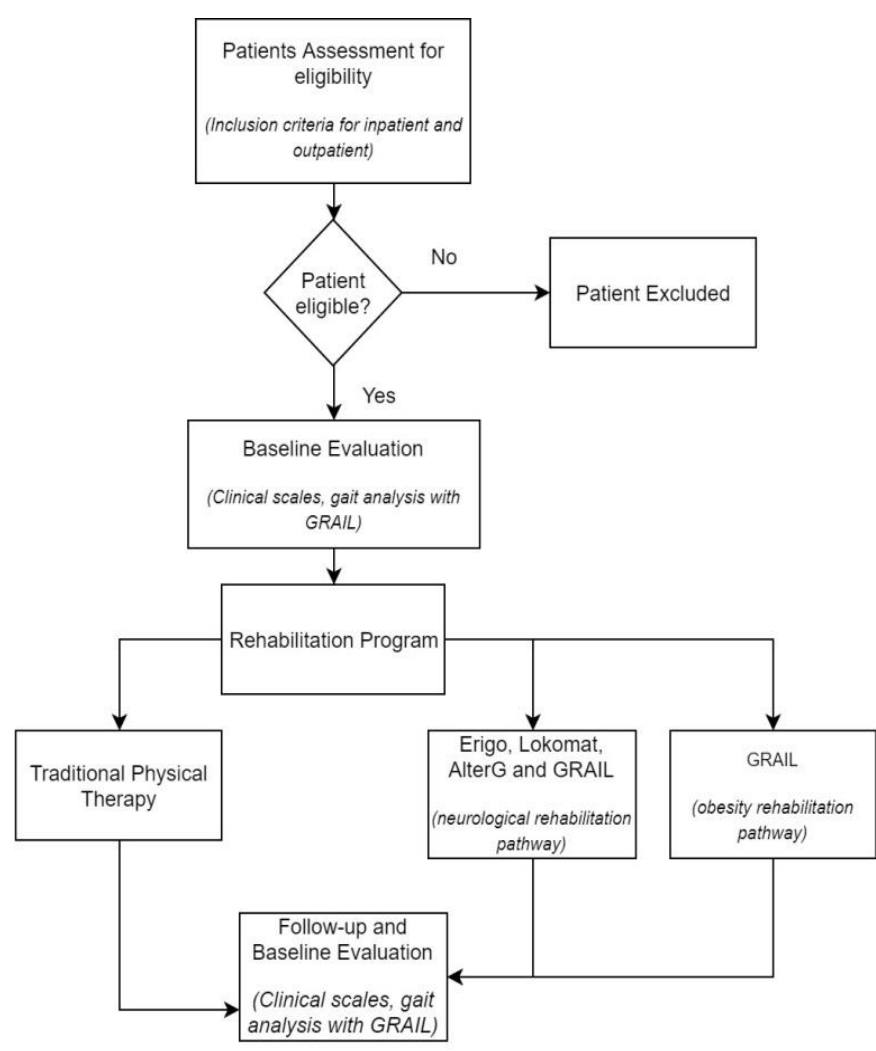

Figure 8. A flowchart showing the patient-based model of technology-based care. The baseline and follow-up evaluations consist of traditional clinical scales (BMI, the Berg Scale, the Barthel Index, etc.) and a GRAIL gait analysis. Depending on their residual motor capability, the participants completed gait training sessions using GRAIL.

\subsection{Rehabilitation pathways}

A flowchart of the implemented technology-based rehabilitation pathways is represented in Figure 8.

The clinical assessments described above were performed at the beginning (baseline) and at the end of an eight-week rehabilitation treatment program (follow-up). The baseline evaluation of all eligible patients consisted of a clinical evaluation performed by the clinicians and physiological measurements of gait patterns recorded by GRAIL. All the spatiotemporal parameters of gait as well as the articular ranges of motion, force, and muscle activation were used as outcome measures together with the clinical scales to assess the baseline state and the effect of the rehabilitation program. Figure 9 shows a representative GRAIL gait analysis report.

The GRAIL rehabilitation sessions, common to both neurological and obesity pathways, consist of a set of different, serious games with the aim of training balance control and dual tasking for patients facing problems in coordination, posture, gait, balance, and/or stability (Figure 10). For example, in the active balance application, the patient is represented as a red ball, and they are required to steer the red ball through the maze by shifting their weight or by flexion, extension, and lateral flexion of the trunk.

The pathway includes up to four hours of individual rehabilitative training for five days a week, which included traditional neuro-motor techniques, articular mobilization, stretching exercises, "sit-to-stand" exercises, and step

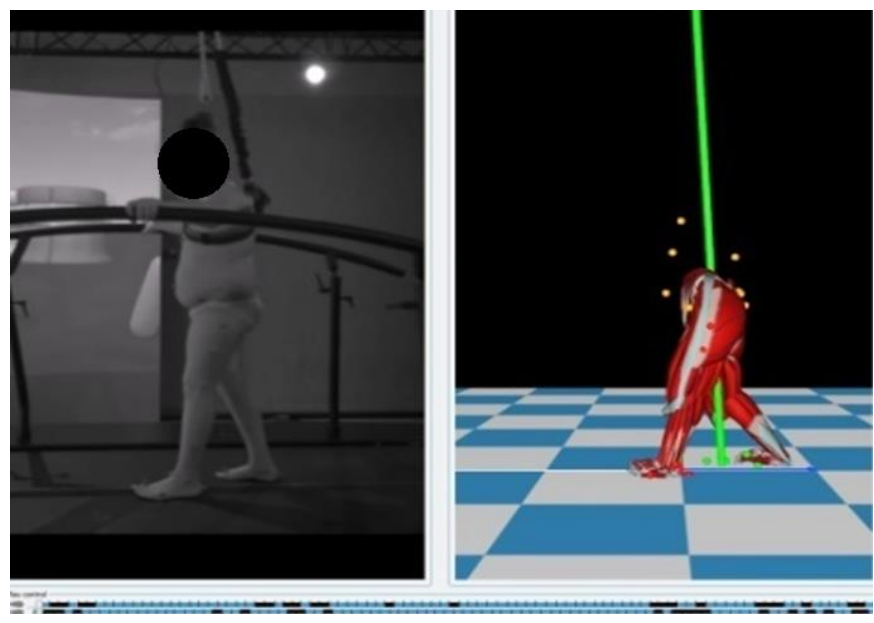

Kinematics - Joint angles - Left
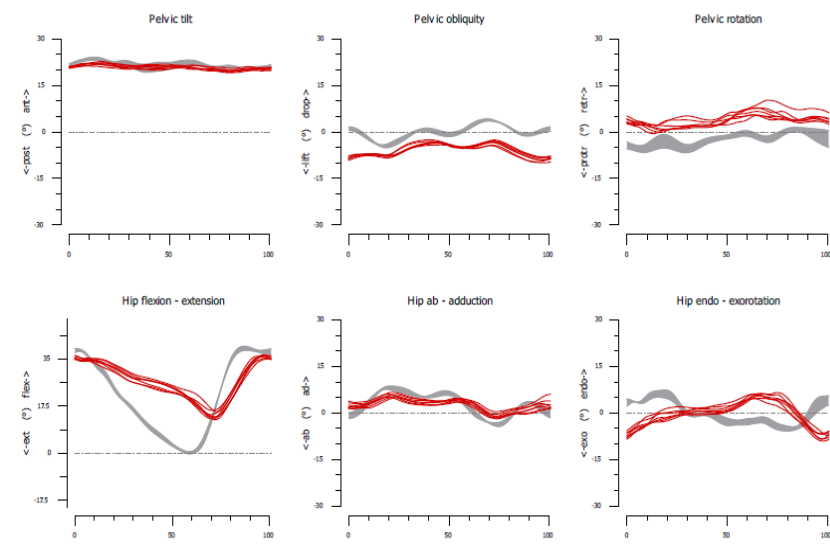

Figure 9. Gait offline tools GRAIL analysis (at the top); an example of such kinematics angles during GRAIL gait analysis sessions. The shaded area denotes normal range (at the bottom). 

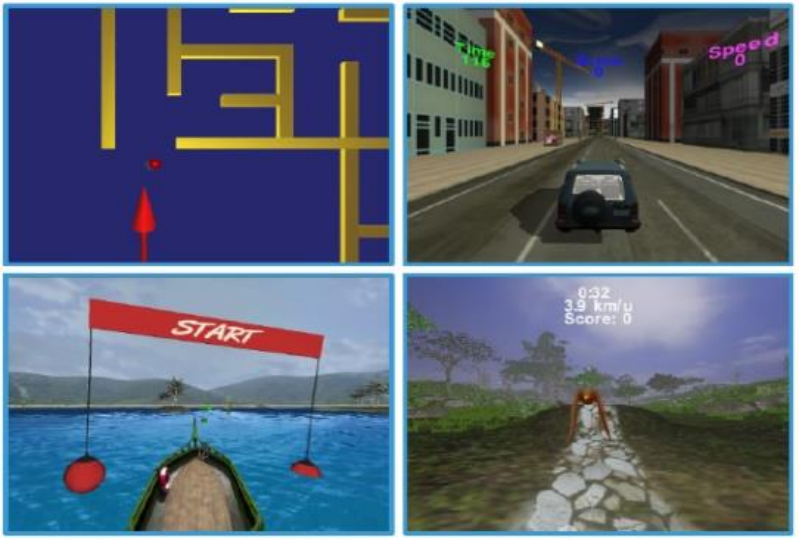

Figure 10. Rehabilitation GRAIL sessions for patients facing problems in coordination, posture, gait, balance, and/or stability.

decomposition exercises. The outpatients receive the same rehabilitation program for about one or two days a week (Day Hospital Rehabilitation).

The neurological rehabilitation pathways include balance and gait training using Erigo, Lokomat, AlterG, and GRAIL. The Erigo device is used for early rehabilitation and mobilizations of the patients, while Lokomat is used for intensive locomotion therapy.

The AlterG allows the patients to undertake gait training on a treadmill with different percentages of body weight support (body weight can be reduced by up to $80 \%$ in $1 \%$ increments), thereby reducing ground reaction forces in the lower limbs and improving balance and walking capacity.

In this way, the system encounters patients both physically and cognitively in realistic, interactive, and controlled environments.

Children interact with the system due to the integrated force plates measuring weight shifting and body motion.

Engagement is further enhanced using realistic sounds and scents. Additionally, GRAIL allows the child to identify problematic balance situations that occasionally occur in everyday life but cannot be replicated in traditional physical therapy.

Measuring the changes in gait pattern during all these conditions allows for the identification and quantification of pathology specific compensation strategies or determining dynamic stability.

Then, clinicians can manipulate the environment interaction conditions, customizing the challenges applying varying levels of difficulty that change automatically according to patient performance, thereby facilitating the learning process in both virtual and real environments.

\section{RESULTS}

Since 2017, a biomedical engineer has participated in the Regional Network for Paediatric Rehabilitation (NETCRIP) to study a rehabilitation assistance network for pediatric patients. His role has been to setup the entire rehabilitation laboratory system and to introduce new technology-based pathways in clinical activities.

In this framework, the enrolment for patients started according to the abovementioned neurological pathways.

To date, over fifty children with gait disorders arising due to neurological diseases have been enrolled in the technology-based rehabilitation program.
Following the encouraging results from this implementation, the obesity rehabilitation pathways have been designed and introduced in clinical activities. To date, the pathway has been tested on one patient.

\section{DISCUSSION}

The primary objective of the current study was to outline a methodology for introducing new robotics and VR systems, such as Erigo, Lokomat, and GRAIL, in patient pathways at the pediatric hospital Santobono-Pausilipon. These technologies are the basis of designing and implementing two technology-based rehabilitation pathways for children with gait disorders arising due to obesity and neurological diseases.

In recent years, several robotics and VR systems for rehabilitation treatments have been developed successfully to help patients with functional disability recover or compensate for loss of limb motor function [14]. These systems allow the patient to perform safe and intensive training that can be done in combination with other treatments.

This is reflected in the opportunities for play that can be enjoyable, stimulating, and non-threatening. Such rehabilitation ensures that patients have access to the professional support, advice, and intervention they need to accomplish their personal rehabilitation targets, maximizing their independence relating to activities of daily living.

The benefit of using VR for rehabilitation is that the type of action, exercise, and feedback can be tailored to the needs and capabilities of the child for more effective motor learning and other rehabilitation outcomes.

However, even if these technologies are available in medical departments, they are usually used only sporadically in gait disorder rehabilitation. This is due to a lack of multidisciplinary teams in clinical practice and to the fact that customized devicebased therapy processes still contrast strongly with typical ways of working in rehabilitation, still oriented towards manual activities (the traditional approach for rehabilitation).

Moreover, given the labor intensiveness of creating technology-based rehabilitation pathways, the use of these tools is still not common in rehabilitation. The use of technologybased custom care pathways can often reduce in-hospital complications and can lead to clear positive effects on safety and quality of care, particularly for children.

The rapid involvement of healthcare professionals in such projects has shown that the design of a new clinical pathway is useful in promoting a patient-centered approach. This is especially true if pathways are technologically driven by new physiological measurements and modeling methods.

Now the operative procedure has been defined, it is now necessary to define robust indices [15] to evaluate the pathway itself and its introduction in clinical activities.

In order to do so, the next step is to analyze the clinical results using a robust statistical analysis in order to investigate and gain feedback on patient acceptance of this new integrated and technology-based clinical pathway

\section{ACKNOWLEDGEMENTS}

This research was supported by Regione Campania - Regional Network for Paediatric Rehabilitation (NETCRIP). We give special thanks to the Management of AORN SantobonoPausilipon for the rapid reception of the proposed rehabilitation clinical pathway. 


\section{REFERENCES}

[1] D. F. Higginbotham, H. Shane, S. Russell, K. Caves, Access to AAC: present, past, and future, Augment. Altern. Commun. 23 (2007) pp. 243-257.

[2] L. Ball, A. Nordness, S. Fager, et al., Eye gaze access of AAC technology for persons with amyotrophic lateral sclerosis, J. Med. Speech Lang. Pathol. 18 (2010) pp. 11-23.

[3] S. Fager, L. Bardach, S. Russell, J. Higginbotham, Access to augmentative and alternative communication: new technologies and clinical decision-making, J. Pediatr. Rehabil. Med. 5 (2012) pp. 53-61.

[4] P. Langhorne, F. Coupar, A. Pollock, Motor recovery after stroke: a systematic review, Lancet Neurol. 8(8) (2009) pp. 741-754.

[5] L. Iuppariello, P. Bifulco, M. Cesarelli, S. Esposito, M. Nespoli, L. Foggia, F. Clemente, "New measurement techniques for gait analysis: the GRAIL experience", Proc. of the $22^{\text {nd }}$ IMEKO TC4 International Symposium, Sept. 14-15, 2017, Iasi, Romania, pp. 249-252.

[6] R. L. O'Neil, R. L. Skeel, K. I. Ustinova, Cognitive ability predicts motor learning on a virtual reality game in patients with TBI, Neurorehabilitation 33(4) (2013) pp. 667-680.

[7] V. D. Tozzi, F. Longo, G. Pacileo, D. Salvatore, N. Pinelli, V. Morando. Patient workflow for chronic care model (in Italian), FIASO - Italian Federation of Health Services, Rome, 2014.

[8] W. Ming et al. "Locomotor training through a 3D cable-driven robotic system for walking function in children with cerebral palsy: a pilot study", Proc. of the $36^{\text {th }}$ Annual International Conference of the IEEE, Aug. 26-30, 2014, Chicago, U.S.A., pp. 3529-3532.
[9] J. A. Day, et al. Locomotor training with partial body weight support on a treadmill in a non-ambulatory child with spastic tetraplegic cerebral palsy: a case report, Pediatric Physical Therapy 16(2) (2004) pp. 106-113.

[10] I. Borggraefe, et al. Robotic-assisted treadmill therapy improves walking and standing performance $\mathrm{n}$ children and adolescents with cerebral palsy. European Journal of Paediatric Neurology 14(6) (2010), pp. 496-502.

[11] I. Borggraefe, et al. Improved gait parameters after roboticassisted locomotor treadmill therapy in a 6 -year-old child with cerebral palsy, Movement Disorders 23(2) (2008) pp. 280-283.

[12] G. K. R. Berntsen, D. B. Gammon, A. Steinsbekk, N. Foss, C. Ruland, V. Fønnebø, What are the goals of care in individual Patient Pathways? An qualitative analysis of how different health concepts may affect goals for care", International Journal of Integrated Care 15(5) (2015) pp. 1-2.

[13] T. Geijtenbeek, F. Steenbrink, B. Otten, O.E.Zohar, "D-flow: immersive virtual reality and real-time feedback for rehabilitation", Proc. of the $10^{\text {th }}$ International Conference on Virtual Reality Continuum and Its Applications in Industry, Dec. 11-12, 2011, Hong Kong, China.

[14] M. Cesarelli, G. D’Angelo, M. Romano, P. Bifulco, F. Clemente, "A new system to deliver vibration, measure motion and EMG reaction for forearm muscles", 20th IMEKO TC4 Symposium on Measurements of Electrical Quantities and the $18^{\text {th }}$ TC4 International Workshop on ADC and DCA Modeling and Testing, IWADC, Sept. 15-17, 2014, Benevento, Italy, pp. 606609.

[15] F. Clemente, M. D'Arco, E. D'Avino. The use of a conceptual model and related indicators to evaluate quality of healthcare, in intensive care units", Qual. Eng. 26, (2014) pp. 196-205. 